\title{
Plexin A-Semaphorin-1a Reverse Signaling Regulates Photoreceptor Axon Guidance in Drosophila
}

\author{
Li Yu, ${ }^{1}$ Yating Zhou, ${ }^{2}$ Songting Cheng, ${ }^{1}$ and Yong Rao ${ }^{1}$ \\ ${ }^{1}$ McGill Centre for Research in Neuroscience, Department of Neurology and Neurosurgery, Department of Medicine, and ${ }^{2}$ Department of Biology, McGill \\ University Health Centre, Montreal, Quebec H3G 1A4, Canada
}

While it is well established that Semaphorin family proteins function as axon guidance ligands in invertebrates and vertebrates, several recent studies indicate that the Drosophila Semaphorin-1a (Semala), a transmembrane Semaphorin, can also function as a receptor during neural development. The regulator of Semala reverse signaling, however, remains unknown. In this study, we show that like Semala, the well known Semaphorin receptor Plexin A (PlexA), is required for the proper guidance of photoreceptor (R cell) axons in the Drosophila visual system. Loss of PlexA, like loss of semala, disrupted the association of R-cell growth cones in the optic lobe. Conversely, overexpression of PlexA, like overexpression of semala, induced the hyperfasciculation of R-cell axons. Unlike Semala, however, the cytoplasmic domain of PlexA is dispensable. Epistasis analysis suggests that PlexA functions upstream of semala. And PlexA and sema1a interact genetically with Rho1. We propose that PlexA regulates Semala reverse signaling in the Drosophila visual system.

\section{Introduction}

Neuronal growth cones respond to cues present in the surrounding environment in guiding axons toward their target region (Tessier-Lavigne and Goodman, 1996). The Semaphorin family proteins are well known repulsive guidance cues (Tamagnone and Comoglio, 2000; Pasterkamp and Kolodkin, 2003), while some Semaphorins can also induce attractive responses (Wong et al., 1999; Polleux et al., 2000; Dalpé et al., 2005). The action of Semaphorins is mediated by two families of growth-cone receptors, plexins and neuropilins (Fujisawa and Kitsukawa, 1998). Several cell surface receptor proteins such as Off-track (Otk) (Winberg et al., 2001), L1 (Castellani et al., 2000), Gyc76C (Ayoob et al., 2004), heparan sulfate proteoglycans and chondroitin sulfate proteoglycans (Kantor et al., 2004), have been shown to function as part of the receptor complex for Semaphorins in axon guidance.

Recent studies on the Drosophila transmembrane Semala demonstrate that Semaphorin can also function as a receptor (for review, see Zhou et al., 2008). Semala was originally identified as a repulsive ligand in mediating motor axon guidance in the fly embryo (Yu et al., 1998). Semala binds to its receptor Plexin A (PlexA) (Winberg et al., 1998), which forms a complex with the

Received March 22, 2010; revised April 20, 2010; accepted July 27, 2010.

This work was supported by an operating grant (MOP-14688) awarded to Yong Rao from Canadian Institutes of Health Research. Y.R. is a Le Fonds de la Recherche en Santé de Québec senior research scholar. Y.L. is a recipient of Le Fonds de la Recherche en Santé de Québec studentship. We thank people in the laboratories of Yong Rao and Don van Meyel for comments and suggestions; the Bloomington Stock Center for fly stocks; the Developmental Studies Hybridoma Bank at University of lowa for MAb 24B10; Liqun Luo for anti-PlexA antibody and pMF3-UAS-PlexA-RNAi transgenic line; Corey Goodman for UAS-sema 1a; and Alex Kolodkin for sema $1 a^{\text {P1 }}$ and UAS-PlexA.

Correspondence should be addressed to Yong Rao, Centre for Research in Neuroscience, The Montreal General Hospital Research Institute, Room L7-136, 1650 Cedar Avenue, Montreal, Quebec H3G 1A4, Canada. E-mail: yong.rao@mcgill.ca.

DOI:10.1523/JNEUROSCI.1494-10.2010

Copyright $\odot 2010$ the authors $\quad$ 0270-6474/10/3012151-06\$15.00/0 receptor tyrosine kinase Otk (Winberg et al., 2001) and regulates the flavoprotein monooxygenase MICAL (Terman et al., 2002) and the A kinase anchoring protein Nervy (Terman and Kolodkin, 2004) during motor axon guidance. Our recent work shows that Semala functions as a receptor to regulate photoreceptor ( $\mathrm{R}$ cell) axon guidance (Cafferty et al., 2006). Similarly, Luo and colleagues show that Semala also functions as a receptor to mediate the targeting of dendrites from projection neurons in the olfactory system (Komiyama et al., 2007). It has also been shown that Semala is involved in synaptic formation by mediating bidirectional signaling in the adult giant fiber system (Godenschwege et al., 2002). The identity of the upstream protein that activates Semala reverse signaling in axon guidance, however, remains unclear.

Here we show that PlexA interacts with Semala to regulate R-cell axon guidance. The Drosophila compound eye consists of $\sim 800$ repeating units called ommatidia, each contains eight different R cells (i.e., R1-R8) (Tomlinson and Ready, 1987). During the third-instar larval stage $\mathrm{R}$ cells extend their axons into the optic lobe (Meinertzhagen and Hanson, 1993; Clandinin and Zipursky, 2002; Tayler and Garrity, 2003). After reaching the lamina, R1-R6 growth cones terminate at appropriate topographic locations, where they form close contacts with neighboring growth cones. Whereas R7 and R8 axons pass through the lamina and elaborate a precise topographic map in the medulla.

In this study, we show that PlexA displayed phenotypes similar to that of semala. Epistasis analysis suggests that PlexA functions upstream of semala to mediate the interaction between neighboring R-cell axons for the establishment of appropriate topographic projections in the optic lobe. The cytoplasmic domain of PlexA is dispensable. PlexA and Semala interact genetically with the cytoskeletal regulator Rho1. These results support a role for PlexA as a regulator of Semala reverse signaling to regulate R-cell axon-axon interaction. 


\section{Materials and Methods}

Genetics. UAS-Rho1.N19 flies were obtained from the Bloomington Drosophila Stock Center. To examine PlexA loss-of-function phenotype, genetic crosses were performed to generate PlexA ${ }^{\mathrm{Df}(4) \mathrm{C} 3} /$ GAL4; TM6, Tb/UAS-GFP. Nonfluorescent larvae (genotype: $P \operatorname{lex} A^{\mathrm{Df}(4) \mathrm{C} 3} / P \operatorname{lex} A^{\mathrm{Df}(4) \mathrm{C} 3}$ ) from the resulting line was selected for dissection. To knock down the expression level of PlexA, UAS-PlexA-RNAi (pWIZ) or UAS-PlexA-RNA (pMF3) (provided by L. Luo, Stanford University, Stanford, CA) flies were crossed with GMRGAL4 flies. To overexpress full-length PlexA or cytoplasmic-domain truncated PlexA (i.e., plexA ${ }^{\Delta \mathrm{cyt}}$ ), UAS-PlexA or UAS-PlexA ${ }^{\Delta \mathrm{cyt}}$ flies were crossed with GMR-GAL4 flies. To examine the potential genetic interaction between semala and PlexA, genetic crosses were performed to generate sema1a ${ }^{\mathrm{P} 1} / \mathrm{UAS}-\mathrm{PlexA}-R N A i$; longGMR-GAL4/+. To remove semala in flies overexpressing PlexA, genetic crosses were performed to generate semala $\mathrm{P}^{\mathrm{P} 1}, \mathrm{GMR}-$ GAL4/semala $a^{\mathrm{Df}(2) \mathrm{N} 22-5}, \mathrm{UAS}-$ PlexA. To express UAS-Plex $A^{\Delta \text { cyt }}$ in $\mathrm{R}$ cells in which endogenous PlexA is knocked down, genetic crosses were perform to generate GMR-GAL4/+; UAS$P l e x A^{\Delta \text { cyt }} / \mathrm{UAS}$-PlexA-RNAi (pWIZ). To investigate potential genetic interactions between Semala and a set of intracellular signaling proteins, GMR-GAL4, UAS-sema1a/Bc flies were crossed with flies carrying the mutations, thus reducing the dosage of these genes by $50 \%$ in flies overexpressing semala. To express the dominant-negative form of Rhol (i.e., Rho1.N19) in R cells, genetic crosses were performed to generate GMR-GAL4/UAS-Rho1.N19.

Histology. Dissection, fixation and staining of the eye-brain complexes from third-instar larvae were performed similarly as described previously (Ruan et al., 1999). MAb 24B10 and anti-GFP antibodies were used at 1:100 and 1:1000 dilutions, respectively. The secondary antibodies (Jackson Immunochemicals) were used at 1:200 dilution. Anti-PlexA antibody was used at 1:1000 dilution. Epifluorescent images were captured using a high-resolution fluorescence imaging system (Canberra Packard) and analyzed by two-dimensional deconvolution using MetaMorph imaging software (Universal Imaging).

Molecular biology. To generate GST-PlexA fusion protein for raising anti-PlexA antibody, a 350 bp sequence encoding for amino acid sequence Thr701 to Leu818 in the PlexA extracellular region was amplified by PCR using two primers 5 'cagcgaattcacagctgaaaactgccgg 3 ' and $5^{\prime}$ atgactcgagaagtggcttcgaccc $3^{\prime}$. This fragment was subcloned into the EcoRI and XhoI sites of pGEX-4T-1 vector.

To generate UAS-PlexA-RNAi transgenic construct, a $\sim 600 \mathrm{bp}$ fragment encoding for a portion of PlexA cytoplasmic domain was amplified by PCR using two primers $5^{\prime}$ gccatctagaggagttcgaatagctgg $3^{\prime}$ and $5^{\prime}$ ccgatctagagcttgcatacatctctcc $3^{\prime}$. This fragment was subcloned into the pWIZ vector to generate a construct containing an inversely repeated sequence.

To generate the UAS-PlexA ${ }^{\Delta \text { cyt }}$ construct, two primers $5^{\prime}$ ggtgacaccatttactgcgatagc $3^{\prime}$ and $5^{\prime}$ 'cttgctagcgtagctccagaatgtccatctg $3^{\prime}$ were used to amplify the fragment encoding for the amino acid sequence G785 to R1326 followed by a stop codon TAG by PCR. The PCR fragment was digested by XbaI and NheI, and subsequently used to replace the XbaIXbaI fragment in the full-length UAS-PlexA construct. The resulting plasmid lacks the sequence encoding for almost entire cytoplasmic domain (i.e., amino acid 1327-1945).

\section{Results}

PlexA is required in $\mathrm{R}$ cells for the proper association of $\mathrm{R}$-cell growth cones at the intermediate target region

That Semala functions as a receptor in R-cell axon guidance raises the interesting possibility that PlexA, the well known Semaphorin receptor, activates Semala reverse signaling in R-cell axons. To determine the potential role of PlexA in the developing Drosophila visual system, we performed loss-of-function analysis. While most of mutants homozygous for a deficiency chromosome (i.e., PlexA ${ }^{\mathrm{Df}(4) \mathrm{C} 3}$ ) lacking the PlexA gene died at late embryonic stage, occasionally some mutants could reach the third-instar larval stage, which allowed us to examine the effect of PlexA deletion on R-cell axonal projection pattern.
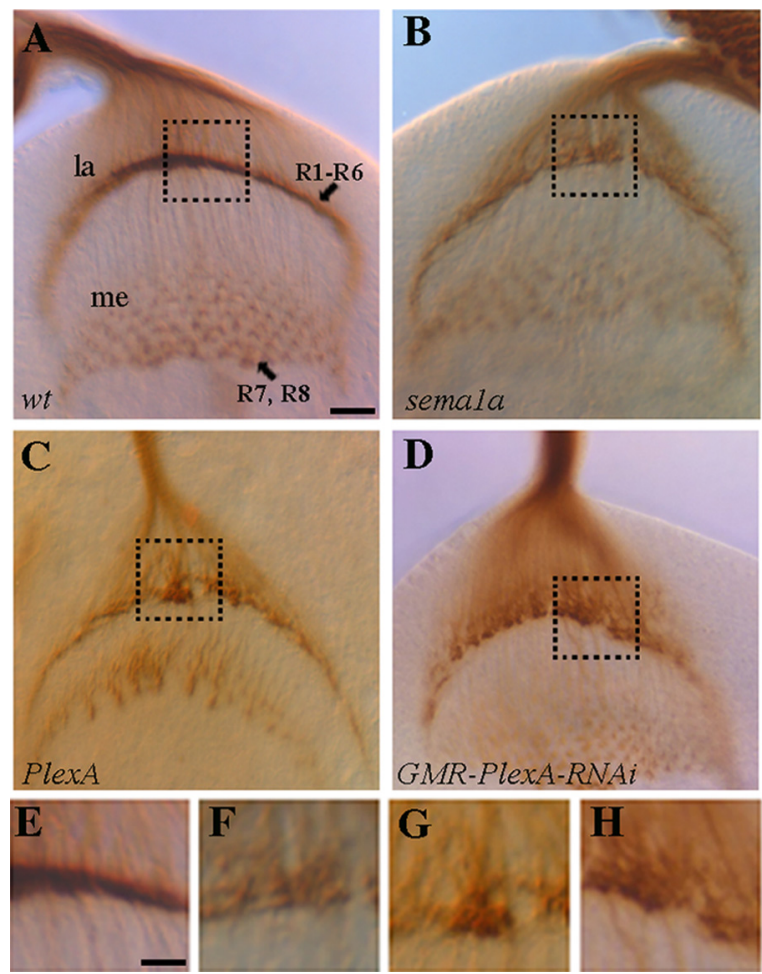

Figure 1. PlexA is required for the proper formation of R1-R6 termination layer in the developing optic lobe. Third-instar larval eye- brain complexes were stained with MAb 24B10 to visualize R-cell axonal projection pattern. $\boldsymbol{A}$, Wild type. R1-R6 axons stop at the intermediate target region in the lamina (la), where their growth cones expand and associate closely with each other to form a smooth and dense terminal layer. Whereas R7 and R8 axons project through the lamina into the medulla (me). $\boldsymbol{B}$, sema $1 a^{\mathrm{P} 1}$ homozygote. R1-R6 terminal layer was disrupted. R1-R6 growth cones associated loosely with neighboring growth cones and failed to pack into a dense layer. $\boldsymbol{C}, \boldsymbol{D}$, A similar phenotype was observed in PlexA deficiency mutants ( $\boldsymbol{C}$ ) and eye-specific PlexA knockdown mutants $(\boldsymbol{D})$. $\boldsymbol{E}-\boldsymbol{H}$ are enlarged views of the boxed regions in $\boldsymbol{A}-\boldsymbol{D}$, respectively. Scale bar: $\boldsymbol{A}-\boldsymbol{D}, 10 \mu \mathrm{m} ; \boldsymbol{E}-\boldsymbol{H}, 5 \mu \mathrm{m}$.

In wild type (Fig. $1 A$ ), the differentiating R-cells project axons through the optic stalk into the developing optic lobe. After migrating over the superficial lamina, R1-R6 axons terminate in between two layers of lamina glial cells, and their growth cones associate closely with each other to form a continuous and dense terminal layer at the intermediate target region. R7 and R8 axons within the same bundle extend through the lamina into the deeper medulla layer.

In our previous study (Cafferty et al., 2006), we showed that Semala functions as a receptor to mediate attractive interactions between R1-R6 growth cones at the lamina intermediate target region. Mutations in the semala gene disrupted the association of $\mathrm{R} 1-\mathrm{R} 6$ growth cones at the intermediate target region, leading to the appearance of a discontinuous termination layer in the lamina, where R1-R6 growth cones scattered around the lamina termination region (Fig. 1B,F). Interestingly, we found that homozygous PlexA mutants displayed a similar phenotype as R1-R6 growth cones failed to form a continuous and dense termination layer in all mutants examined (100\%, $n=9$, Fig. $1 C, G)$.

Our previous study showed that Semala functions in $\mathrm{R}$ cells to mediate the association between R1-R6 growth cones (Cafferty et al., 2006). If PlexA functions in the same pathway, one would predict that PlexA is also required in R cells. Since PlexA is located on the fourth chromosome, it is not feasible to perform FRT-mediated genetic mosaic analysis to determine whether PlexA is also required in $\mathrm{R}$ cells. To circumvent this 

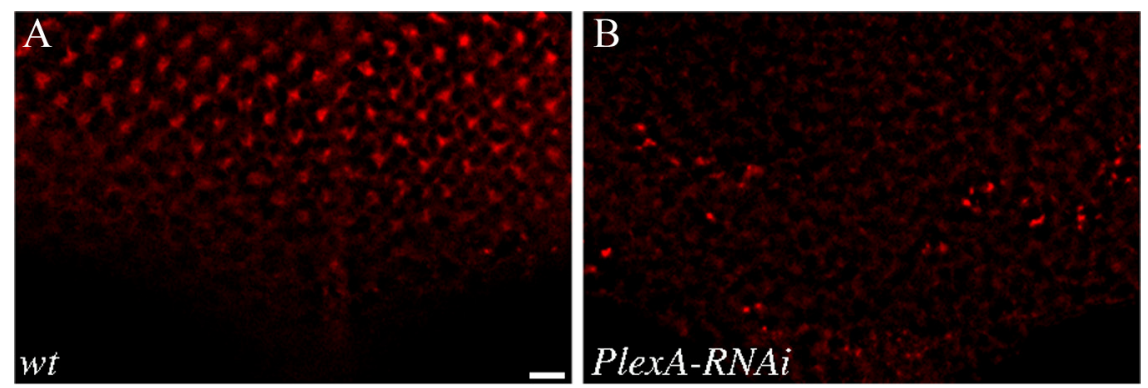

Figure 2. Eye-specific expression of the UAS-PlexA-RNAi transgene effectively decreased the level of PlexA in $\mathrm{R}$ cells. Thirdinstar eye discs were stained with anti-PlexA antibody. $\boldsymbol{A}$, Wild-type. Strong PlexA staining was detected in developing R-cell clusters in the posterior region of the eye disc. $\boldsymbol{B}$, PlexA staining was significantly reduced when the level of PlexA was knocked down by expressing a UAS-PlexA-RNAi transgene under control of the eye-specific GMR-GAL4 driver. Scale bar, $5 \mu \mathrm{m}$.
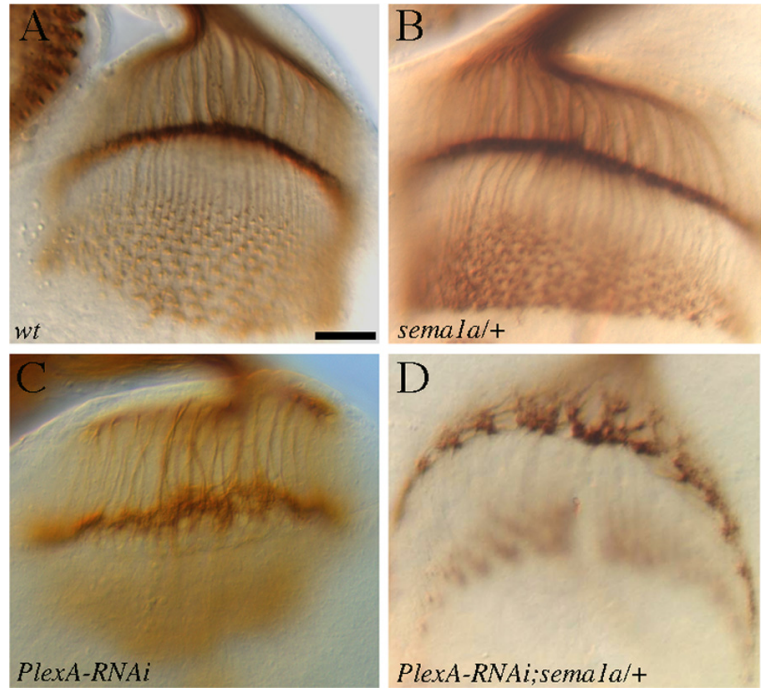

Figure 3. PlexA interacts genetically with sema 1a. R-cell axonal projection pattern in thirdinstar larval eye-brain complexes was visualized with MAb $24 B 10$ staining. $\boldsymbol{A}$, Wild type. $\boldsymbol{B}$, Normal R-cell projection pattern was observed in sema1a heterozygotes. $C$, Larvae in which the level of PlexA was specifically knocked down in the eye displayed a mild phenotype. $\boldsymbol{D}$, Reducing the dosage of sema 1 a by $50 \%$ in PlexA knockdown larvae increased both penetrance and severity of the phenotype. Scale bar, $20 \mu \mathrm{m}$.

problem, we performed gene knockdown analysis. We examined R-cell axonal projection pattern in fly larvae in which the expression of PlexA was specifically knocked down in $\mathrm{R}$ cells by expressing a UAS-PlexA-RNA $i$ transgene (i.e., pWIZ-UASPlexA-RNAi) under control of the eye-specific driver GMRGAL4. Compared with that of PlexA mutants (Fig. 1C,G), eye-specific PlexA knockdown mutants displayed a similar defect at the lamina termination layer $(\sim 20 \%, n=41$; Fig. $1 D, H)$. Similar results were obtained when PlexA was knocked down by using another PlexA-RNAi transgenic construct (pMF3) that targets a different sequence in the PlexA gene $(\sim 19 \%, n=$ 21 ), which was generated by Luo and colleagues (Komiyama et al., 2007).

To confirm that eye-specific expression of UAS-PlexA-RNAi effectively knocked down the level of PlexA, we performed immunohistochemical analysis. Third-instar larval eye discs were stained with a rabbit anti-PlexA antibody. At third-instar larval stage, precursor cells in the eye-imaginal disc begin to differentiate into R cells. In wild type (Fig. 2 A), like Sema la (Cafferty et al., 2006), PlexA is detected in $\mathrm{R}$ cells in the posterior region of the eye disc (Fig. 2A). We found that the level of PlexA staining was significantly reduced when UASPlexA-RNA $i$ was expressed in the developing eye disc (Fig. $2 B$ ).

Thus, like semala, PlexA is also required in $\mathrm{R}$ cells for the proper interaction of R1-R6 growth cones in the intermediate target region.

PlexA interacts genetically with sema1a To determine whether PlexA and semala function in the same pathway, we examined the potential genetic interaction between PlexA and semala. The dosage of semala was reduced by $50 \%$ in PlexA knockdown larvae in which the reduction in PlexA level caused a low-penetrant phenotype. If PlexA and semala function in the same pathway, the prediction is that reducing the dosage of semala would further weaken the pathway leading to the enhancement of the phenotype. Indeed, we found that reducing the dosage of semala by $50 \%$ significantly enhanced the PlexA knockdown phenotype (Fig. 3D). The penetrance of the phenotype was increased from $\sim 20 \%(n=41)$ to $\sim 60 \%(n=25)$, and the severity of the phenotype was also increased (Fig. $3 D$ ). This result is consistent with that PlexA and semala function in the same pathway.

\section{Overexpression of PlexA induced the hyperfasciculation of R-cell axons}

Our previous study showed that Semala functions as a receptor to mediate an attractive interaction between R-cell axons (Cafferty et al., 2006), and overexpression of Semala could induce the hyperfasciculation of R-cell axons (Fig. 4 B) (Cafferty et al., 2006). If PlexA functions in the same pathway, one would predict that overexpression of PlexA should induce a similar phenotype. To address this, we examined R-cell projection pattern in third-instar larvae in which PlexA was overexpressed in R-cell axons under control of the eye-specific driver GMR-GAL4. Like overexpression of semala (Fig. $4 B$ ), we found that overexpression of PlexA also induced R-cell axonal hyperfasciculation in all hemispheres examined $(n=20$, Fig. $4 C)$. Compared with wild type (Fig. 4A), overexpression of semala (Fig. $4 B$ ) or PlexA (Fig. 4C) caused the formation of thicker axonal bundles, which led to the decrease in the number of separate axonal bundles between lamina and medulla. Unlike that of wild type in which $\mathrm{R}$-cell axons form a precise array of separate and expanded "Y"-shape terminal structure in the medulla (Fig. $4 A$ ), most of R-cell axons in larvae ovexpressing semala (Fig. $4 B$ ) or PlexA (Fig. $4 C$ ) fused with each other to form large clumps in the medulla.

The cytoplasmic domain of PlexA is dispensable for inducing R-cell axonal hyperfasciculation

Our previous study showed that the cytoplasmic domain of Semala is essential for its function in R-cell axon guidance (Cafferty et al., 2006). Similarly, Luo and colleagues showed that the function of Semala in the olfactory system also requires its cytoplasmic domain (Komiyama et al., 2007). To further determine the action of PlexA in R-cell axon guidance, we examined whether the cytoplasmic domain of PlexA is necessary for its action in R-cell axon guidance.

We generated a UAS-PlexA ${ }^{\Delta \text { cyt }}$ transgene in which the cytoplasmic domain of PlexA was deleted. This transgene was overexpressed in R-cell axons under control of the eye-specific 
GMR-GAL4 driver. Interestingly, we found that like full-length PlexA (Fig. 5B), $P \operatorname{lex} A^{\Delta \text { cyt }}$ was also able to induce the hyperfasciculation of R-cell axons (100\%, $n=21$ hemispheres, Fig. $5 C$ ). We then tested whether expression of PlexA $A^{\Delta \text { cyt }}$ is able to rescue the phenotype induced by PlexA RNAi treatment. PlexA ${ }^{\Delta \text { cyt }}$ was expressed in $\mathrm{R}$ cells in which endogenous PlexA was knocked down by the PlexA RNAi transgene targeting the sequence encoding a portion of the cytoplasmic domain. We found that expression of PlexA ${ }^{\Delta \text { cyt }}$ largely restored the R1-R6 ter-

mination pattern in the lamina (Fig. $5 F, n=39$ ). These results are in marked contrast to the essential requirement of the Semala cytoplasmic domain (Cafferty et al., 2006), and argue against that PlexA functions as a receptor in R-cell axon guidance.

\section{Loss of semala suppressed the PlexA overexpression phenotype}

To further determine the functional relationship between PlexA and sema la in R-cell axon guidance, we performed epistasis analysis. Since overexpression of PlexA induced R-cell axonal hyperfasciculation, we tested whether loss of semala could modify this phenotype. Interestingly, we found that loss of semala largely suppressed the PlexA-induced R-cell axonal hyperfasciculation phenotype (Fig. $6 C, D$ ). This result suggests that PlexA functions upstream of Semala in the pathway, and consistent with that PlexA regulates Semala reverse signaling in $\mathrm{R}$-cell axon guidance.

\section{Sema 1a interacts genetically with Rho1}

To gain further insights into the mechanism of Semala reverse signaling, we examined the potential genetic interaction between Semala and a number of intracellular signaling proteins (supplemental Table 1, available at www.jneurosci.org as supplemental material). Among them, we found that reducing the dosage of small GTPase Rhol by $50 \%$ significantly enhanced the Semalaoverexpression-induced hyperfasciculation phenotype (Fig. 7C; supplemental Table 1, available at www.jneurosci.org as supplemental material). Whereas reducing the dosage of other members of Rho family small GTPases such as Cdc42 and Rac did not show any obvious effect (supplemental Table 1, available at www.jneurosci.org as supplemental material). A previous study showed that a putative Enabled (Ena)-binding motif in the cytoplasmic domain of Semala is essential for its function in synaptic formation in the adult giant fiber system (Godenschwege et al., 2002). However, we did not detect any obvious interaction between Semala and Ena or between Semala and Abl (Fig. $7 B$; supplemental Table 1, available at www.jneurosci.org as supplemental material).

To further investigate the role of Rho1, we examined whether interfering with the function of Rhol affects R-cell axonal projections. A dominant-negative form of Rho1 (i.e., Rho1.N19) was expressed in R cells under control of the GMR-GAL4 driver. Interestingly, we found that eye-specific expression of Rhol.N19 induced an axonal-hyperfasiculation phenotype similar to overexpression of Semala $(\sim 55 \%, n=20$, Fig. $7 E)$. We then examined whether PlexA, like semala, interacts genetically with Rho1. PlexA was knocked down by RNAi in flies expressing Rho1.N19. We found that the severity of the Rho1.N19-induced hyperfasciculation phenotype was significantly suppressed by PlexA$R N A i$ treatment $(n=23$, Fig. $7 F)$. These results support that
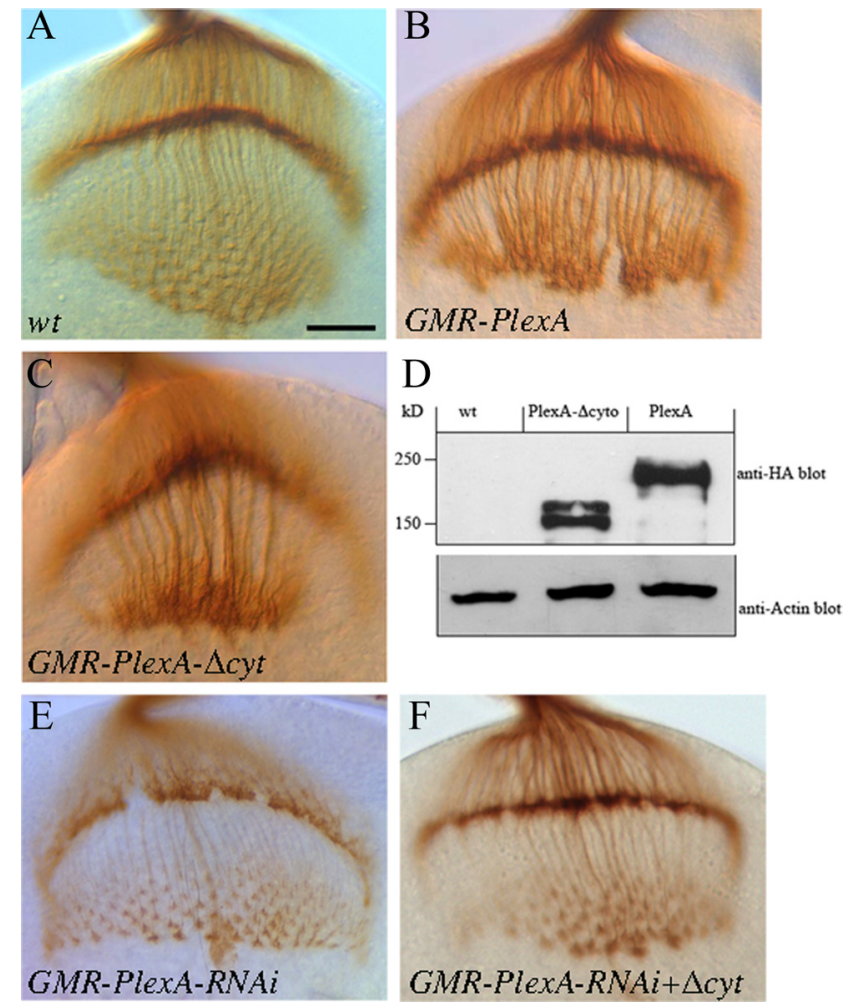

Figure 5. The cytoplasmic domain of PlexA is dispensable. $\boldsymbol{A}$, Wild type. $\boldsymbol{B}, 0$ verexpression of PlexA under control of GMR-GAL4 caused the formation of thicker axonal bundles. C, Overexpression of PlexA ${ }^{\Delta \text { cyt }}$ driven by GMR-GAL4 caused an identical hyperfasciculation phenotype. $D$, Western blot analysis using anti-HA antibody showed the expression of HA-tagged fulllength and cytoplasmic-domain-truncated PlexA in flies under control of GMR-GAL4. The size of transgenic proteins is consistent with the predicted size. $\boldsymbol{E}$, An eye-specific PlexA knockdown mutant. $\boldsymbol{F}$, Expression of PlexA ${ }^{\Delta \text { cyt }}$ largely restored the R1-R6 growth-cone organization pattern in the lamina in PlexA knockdown mutants. Scale bar, $20 \mu \mathrm{m}$.

PlexA-Semala reverse signaling regulates the function of Rhol in $\mathrm{R}$-cell axon guidance.

\section{Discussion}

Recent studies indicate that Semala, the Drosophila transmembrane Semaphorin, can function as a receptor or a component of a receptor complex to mediate axon/dendrite guidance and synaptic formation (Godenschwege et al., 2002; Cafferty et al., 2006; Komiyama et al., 2007). However, it is unclear what molecules activate Semala to trigger downstream signaling events. In this study, we provide genetic evidence to support that PlexA functions as a regulator of Semala reverse signaling in the Drosophila visual system. First, PlexA and semala mutants displayed similar loss-of-function and gain-of-function phenotypes. Second, 

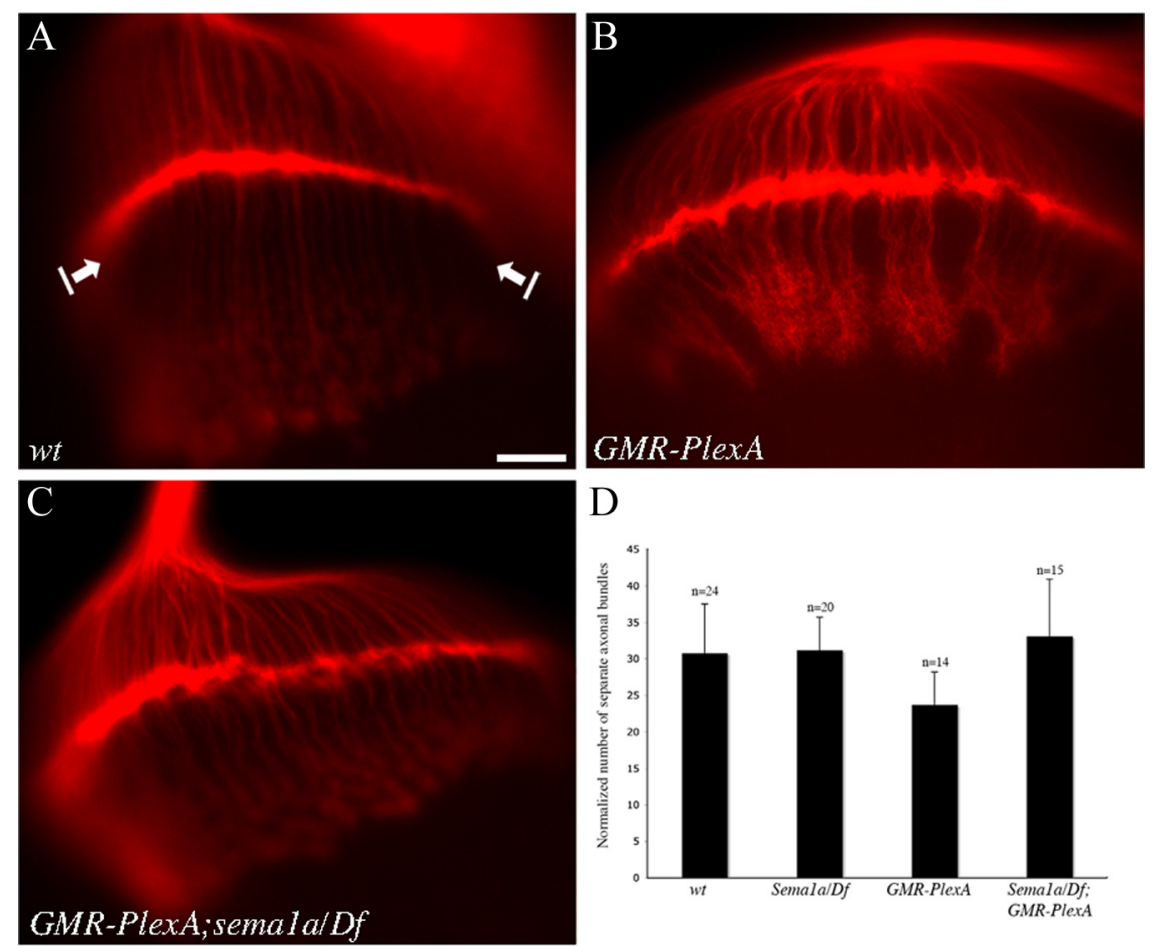
D

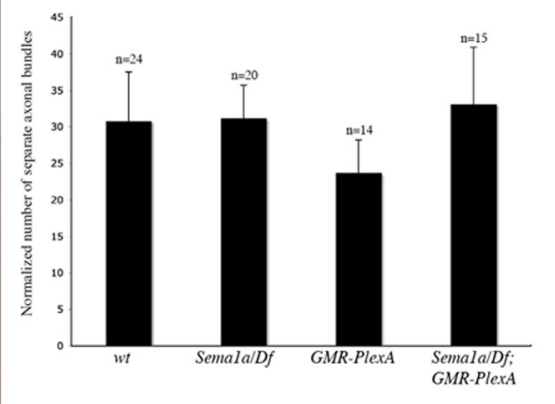

Figure 6. PlexA functions upstream of sema1a. $\boldsymbol{A}$, Wild type. $\boldsymbol{B}$, Overexpression of PlexA under control of GMR-GAL4-induced R-cell axonal hyperfasciculation. C, The PlexA-induced hyperfasciculation phenotype was suppressed when sema $1 a$ was disrupted. $\boldsymbol{D}$, The number of separate axonal bundles that are located between lamina and medulla was counted. The data were normalized with the row number of R-cell clusters in the eye disc. Compared with wild type, overexpression of PlexA induced the formation of thicker bundles and thus significantly decreased the number of separate R-cell axonal bundles ( $p=0.0012$ ). Compared with that of PlexA overexpression in wild-type background, the number of separate axonal bundles in PlexA-overexpression mutants in which the semala gene was disrupted, was increased significantly ( $p=0.0005$ ). Error bars denote SE. Scale bar, $20 \mu \mathrm{m}$. which the interaction between PlexA and Semala induce axon-axon repulsion. First, loss of PlexA, like loss of Semala, appeared to disrupt R-cell axon association leading to the formation of discontinuous termination layer. And second, like overexpression of Semala, overexpression of PlexA induced a R-cell axonal hyperfasciculation phenotype. Since both PlexA and Semala are expressed and genetically required in R cells, we favor a model in which PlexA interacts with Semala to mediate attractive axon-axon interaction for the proper organization of R-cell growth cones at the intermediate target region.

What is the nature of Semala-dependent downstream signaling in R-cell axons? Previous study identified a putative Enabled (Ena)-binding motif in the cytoplasmic domain of Semala that is essential for its function in synaptic formation in the adult giant fiber system (Godenschwege et al., 2002). In vertebrates, it has been shown that some transmembrane Semaphorins use their cytoplasmic domain to recruit intracellular signaling proteins such as EVL (Ena/Vasp-like protein) (Klostermann et al., 2000), PSD-95 (Inagaki et al., 2001; Ohoka et al., 2001; Schultze et al., 2001), c-Src (Eckhardt et al., 1997), Abl kinase and Enabled (Toyofuku et al., 2004). Among them, Abl kinase and Enabled are key components of the Semaphorin6D reverse sigsemala displayed dosage-sensitive genetic interaction with PlexA, consistent with that they function in the same pathway. Third, expression of PlexA ${ }^{\Delta \text { cyt }}$ mutant lacking the cytoplasmic domain was still able to induce R-cell axon hyperfasciculation, and largely rescued the PlexA-RNAi phenotype. And fourth, epistasis analysis suggests that PlexA functions upstream of Semala.

Our results suggest that in addition to functioning in the classic Sema1a-to-PlexA signaling pathway (i.e., Forward signaling) in motor axon guidance (Winberg et al., 1998; Yu et al., 1998), PlexA and its cognate ligand Semala are also capable of mediating PlexA-to-Semala reverse signaling to regulate R-cell axon guidance in the fly visual system. Such ligand/receptor bidirectional signaling is not unprecedented. For instance, it has been shown that Eph receptor tyrosine kinases and their ligands ephrins exhibit both forward and reverse signaling in neural development (Murai and Pasquale, 2003; Davy and Soriano, 2005). Recent studies also indicate a role for ErbB receptors and their ligands Neuregulins bidirectional signaling in neural development (Chen et al., 2008). Such bidirectional signaling mediated by a ligand and receptor complex greatly increases the plasticity of intercellular communications during neural development.

We propose that PlexA interacts with Semala to regulate the communication between neighboring R1-R6 growth cones, which is necessary for the establishment of an appropriate retinotopic termination pattern at the intermediate target region in the developing lamina. Previous studies show that the Semalato-PlexA forward signaling pathway mediates axon-axon repulsion in motor axon guidance (Winberg et al., 1998; Yu et al., 1998) and the targeting of olfactory receptor neurons (Sweeney et al., 2007). However, our results are not consistent with a model in naling pathway for regulating the migration of myocardial cells in the chick embryo (Toyofuku et al., 2004). However, we could not detect any genetic interaction between Semala and Enabled in R-cell axon guidance (supplemental Table 1, available at www.jneurosci. org as supplemental material). Instead, we found that Semala and PlexA interact genetically with small GTPase Rhol (Fig. 7; supplemental Table 1, available at www.jneurosci.org as supplemental material), raising the interesting possibility that Semala reverse signaling involves negative regulation of Rhol. One attractive model is that Semala, activated by PlexA, downregulates the function of Rho1. A decrease in Rhol function may prevent Rhol from inhibiting the function of certain cell adhesion molecules, thus promoting the attractive interaction between R1-R6 growth cones at the intermediate target region. Future studies will be necessary to test this model and elucidate the exact downstream signaling events activated by the PlexA and Semala interaction in the fly visual system.

\section{References}

Ayoob JC, Yu HH, Terman JR, Kolodkin AL (2004) The Drosophila receptor guanylyl cyclase Gyc76C is required for semaphorin-1a-plexin A-mediated axonal repulsion. J Neurosci 24:6639-6649.

Cafferty P, Yu L, Long H, Rao Y (2006) Semaphorin-1a functions as a guidance receptor in the Drosophila visual system. J Neurosci 26:3999-4003.

Castellani V, Chédotal A, Schachner M, Faivre-Sarrailh C, Rougon G (2000) Analysis of the L1-deficient mouse phenotype reveals cross-talk between Sema3A and L1 signaling pathways in axonal guidance. Neuron 27:237-249.

Chen Y, Fu AK, Ip NY (2008) Bidirectional signaling of ErbB and Eph receptors at synapses. Neuron Glia Biol 4:211-221.

Clandinin TR, Zipursky SL (2002) Making connections in the fly visual system. Neuron 35:827-841.

Dalpé G, Brown L, Culotti JG (2005) Vulva morphogenesis involves attrac- 

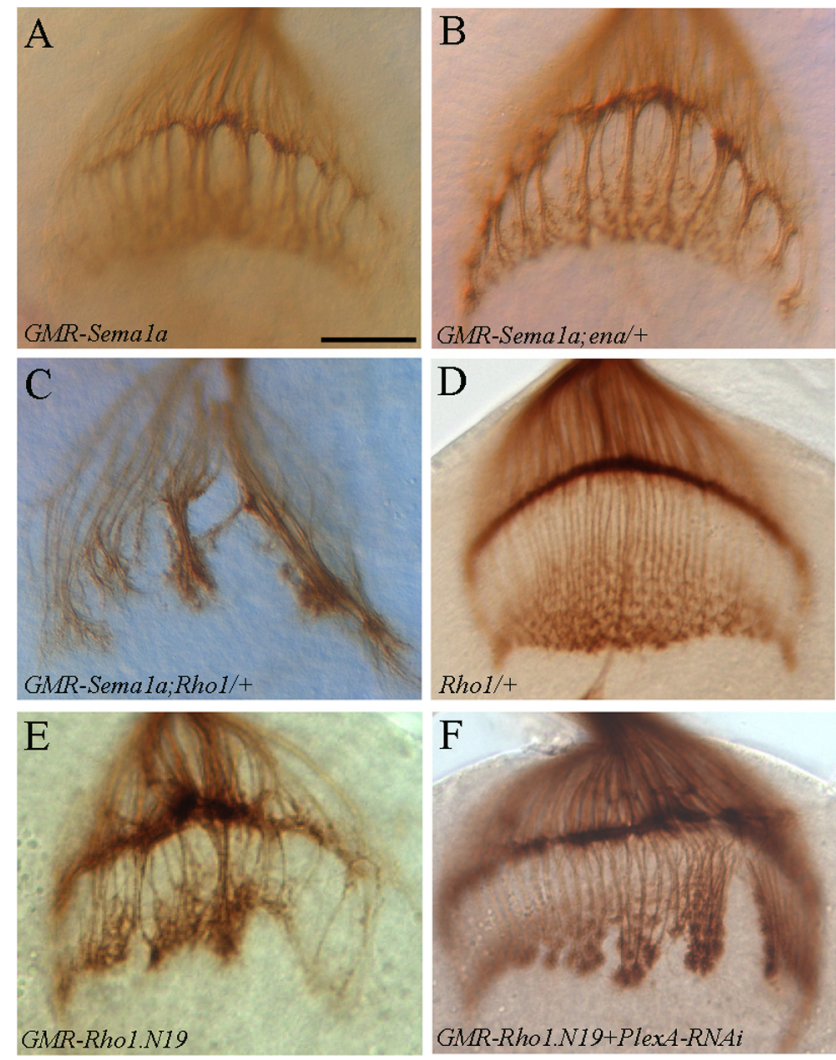

Figure 7. Sema1a and PlexA interact genetically with Rho1. $A$, Overexpression of Sema1a induced the formation of thicker axonal bundles. $\boldsymbol{B}$, Reducing the dosage of ena by half did not modify the Sema1a-induced hyperfasciculation phenotype. $C$, Reducing the dosage of Rho 1 by half significantly enhanced the Sema1a-overexpression phenotype. $\boldsymbol{D}$, Reducing the dosage of $R$ Ro 1 by half in wild-type background did not affect R-cell projection pattern. $\boldsymbol{E}$, Expressing the dominant-negative form Rho1.N19 in R cells also induced an axonal hyperfasciculation phenotype. $\boldsymbol{F}$, Knocking down PlexA suppressed the Rho1.N19-induced phenotype. Scale bar, $20 \mu \mathrm{m}$.

tion of plexin 1-expressing primordial vulva cells to semaphorin 1a sequentially expressed at the vulva midline. Development 132:1387-1400.

Davy A, Soriano P (2005) Ephrin signaling in vivo: look both ways. Dev Dyn 232:1-10.

Eckhardt F, Behar O, Calautti E, Yonezawa K, Nishimoto I, Fishman MC (1997) A novel transmembrane semaphorin can bind c-src. Mol Cell Neurosci 9:409-419.

Fujisawa H, Kitsukawa T (1998) Receptors for collapsin/semaphorins. Curr Opin Neurobiol 8:587-592.

Godenschwege TA, Hu H, Shan-Crofts X, Goodman CS, Murphey RK (2002) Bi-directional signaling by Semaphorin 1a during central synapse formation in Drosophila. Nat Neurosci 5:1294-1301.

Inagaki S, Ohoka Y, Sugimoto H, Fujioka S, Amazaki M, Kurinami H, Miyazaki N, Tohyama M, Furuyama T (2001) Sema4c, a transmembrane semaphorin, interacts with a post-synaptic density protein, PSD95. J Biol Chem 276:9174-9181.

Kantor DB, Chivatakarn O, Peer KL, Oster SF, Inatani M, Hansen MJ, Flanagan JG, Yamaguchi Y, Sretavan DW, Giger RJ, Kolodkin AL (2004) Semaphorin $5 \mathrm{~A}$ is a bifunctional axon guidance cue regulated by heparan and chondroitin sulfate proteoglycans. Neuron 44:961-975.

Klostermann A, Lutz B, Gertler F, Behl C (2000) The orthologous human and murine semaphorin $6 \mathrm{~A}-1$ proteins (SEMA6A-1/Sema6A-1) bind to the enabled/vasodilator-stimulated phosphoprotein-like protein (EVL) via a novel carboxyl-terminal zyxin-like domain. J Biol Chem 275:3964739653.

Komiyama T, Sweeney LB, Schuldiner O, Garcia KC, Luo L (2007) Graded expression of semaphorin-1a cell-autonomously directs dendritic targeting of olfactory projection neurons. Cell 128:399-410.

Meinertzhagen IA, Hanson TE (1993) The development of the optic lobe. In: The development of Drosophila melanogaster (Bate M, Martinez-Arias A, eds), pp 1363-1491. Cold Spring Harbor, NY: Cold Spring Harbor.

Murai KK, Pasquale EB (2003) 'Eph'ective signaling: forward, reverse and crosstalk. J Cell Sci 116:2823-2832.

Ohoka Y, Hirotani M, Sugimoto H, Fujioka S, Furuyama T, Inagaki S (2001) Semaphorin 4C, a transmembrane semaphorin, [corrected] associates with a neurite-outgrowth-related protein, SFAP75. Biochem Biophys Res Commun 280:237-243.

Pasterkamp RJ, Kolodkin AL (2003) Semaphorin junction: making tracks toward neural connectivity. Curr Opin Neurobiol 13:79-89.

Polleux F, Morrow T, Ghosh A (2000) Semaphorin 3A is a chemoattractant for cortical apical dendrites. Nature 404:567-573.

Ruan W, Pang P, Rao Y (1999) The SH2/SH3 adaptor protein dock interacts with the Ste20-like kinase misshapen in controlling growth cone motility. Neuron 24:595-605.

Schultze W, Eulenburg V, Lessmann V, Herrmann L, Dittmar T, Gundelfinger ED, Heumann R, Erdmann KS (2001) Semaphorin4F interacts with the synapse-associated protein SAP90/PSD-95. J Neurochem 78:482489 .

Sweeney LB, Couto A, Chou YH, Berdnik D, Dickson BJ, Luo L, Komiyama T (2007) Temporal target restriction of olfactory receptor neurons by Semaphorin-1a/PlexinA-mediated axon-axon interactions. Neuron 53:185-200.

Tamagnone L, Comoglio PM (2000) Signalling by semaphorin receptors: cell guidance and beyond. Trends Cell Biol 10:377-383.

Tayler TD, Garrity PA (2003) Axon targeting in the Drosophila visual system. Curr Opin Neurobiol 13:90-95.

Terman JR, Kolodkin AL (2004) Nervy links protein kinase a to plexinmediated semaphorin repulsion. Science 303:1204-1207.

Terman JR, Mao T, Pasterkamp RJ, Yu HH, Kolodkin AL (2002) MICALs, a family of conserved flavoprotein oxidoreductases, function in plexinmediated axonal repulsion. Cell 109:887-900.

Tessier-Lavigne M, Goodman CS (1996) The molecular biology of axon guidance. Science 274:1123-1133.

Tomlinson A, Ready DF (1987) Cell fate in the Drosophila ommatidium. Dev Biol 123:264-275.

Toyofuku T, Zhang H, Kumanogoh A, Takegahara N, Yabuki M, Harada K, Hori M, Kikutani H (2004) Guidance of myocardial patterning in cardiac development by Sema6D reverse signalling. Nat Cell Biol 6:12041211.

Winberg ML, Noordermeer JN, Tamagnone L, Comoglio PM, Spriggs MK, Tessier-Lavigne M, Goodman CS (1998) Plexin A is a neuronal semaphorin receptor that controls axon guidance. Cell 95:903-916.

Winberg ML, Tamagnone L, Bai J, Comoglio PM, Montell D, Goodman CS (2001) The transmembrane protein Off-track associates with Plexins and functions downstream of Semaphorin signaling during axon guidance. Neuron 32:53-62.

Wong JT, Wong ST, O'Connor TP (1999) Ectopic semaphorin-1a functions as an attractive guidance cue for developing peripheral neurons. Nat Neurosci 2:798-803.

Yu HH, Araj HH, Ralls SA, Kolodkin AL (1998) The transmembrane Semaphorin Sema I is required in Drosophila for embryonic motor and CNS axon guidance. Neuron 20:207-220.

Zhou Y, Gunput RA, Pasterkamp RJ (2008) Semaphorin signaling: progress made and promises ahead. Trends Biochem Sci 33:161-170. 